Montgomery-Asberg Depression Rating Scale (MADRS) and Hamilton Anxiety Rating Scale (HAM-A). RA-pts with ADD were divided into the following treatment groups: 1 - cDMARDs ( $n=39), 2-$ cDMARDs + PPT (sertraline or mianserine) $(n=43), 3-c D M A R D s+$ bDMARDs $(n=32), 4-c D M A R D s+$ bDMARDs + PPT (sertraline or mianserine) $(n=9)$. Biologics treatment duration varied from 1 to 6 years, antidepressants - from 6 to 96 weeks.

Results: $\mathrm{HAQ}$ scores were high in all 4 groups at baseline and after five years remained high in all groups except group 2 with the lowest endpoint scores among 4 groups (table 1). To measure changes in HAQ scores between groups we compared the differences between baseline and endpoint $\mathrm{HAQ}$ scores $(\triangle \mathrm{HAQ}=$ endpoint $\mathrm{HAQ}$ - baseline $\mathrm{HAQ}$ ) (table 2). The table shows an improvement in HAQ scores in groups 2 and 3 , no significant changes in group 4 and a worsening of HAQ scores in group 1. $\mathrm{HAQ}$ scores in groups 2 and 3 significantly improved compared to group 1.

Table 1 Mean HAQ scores in RA patients with ADD at baseline and after 5 years, by groups.

\begin{tabular}{lccc}
\hline Groups & $\begin{array}{c}\text { At baseline } \\
(\mathrm{n}=128)\end{array}$ & $\begin{array}{c}\text { After } 5 \text { years } \\
(\mathrm{n}=83)\end{array}$ & $\begin{array}{c}\text { P between } \\
\text { time points }\end{array}$ \\
\hline 1 (cDMARDs), $\mathrm{n}=39$ & $1,39 \pm 0,75$ & $1,61 \pm 0,7$ & $>0,05$ \\
2 (cDMARDs + PPT), $\mathrm{n}=43$ & $1,42 \pm 0,9$ & $0,85 \pm 0,66$ & 0,011 \\
3 (cDMARDs + bDMARDs), $\mathrm{n}=32$ & $1,58 \pm 0,76$ & $1,36 \pm 0,71$ & $>0,05$ \\
4 (cDMARDs + bDMARDs + PPT), $\mathrm{n}=9$ & $1,38 \pm 0,83$ & $1,49 \pm 0,26$ & $>0,05$ \\
P between groups & $>0,05$ & $\mathrm{P}_{2-1}<0,001 ;$ & \\
& & $\mathrm{P}_{2-3}^{2}=0,023$ & \\
& & $\mathrm{P}_{2-4}=0,015$ & \\
\hline
\end{tabular}

Table 2. Differences between baseline and 5-years endpoint HAQ scores, by groups

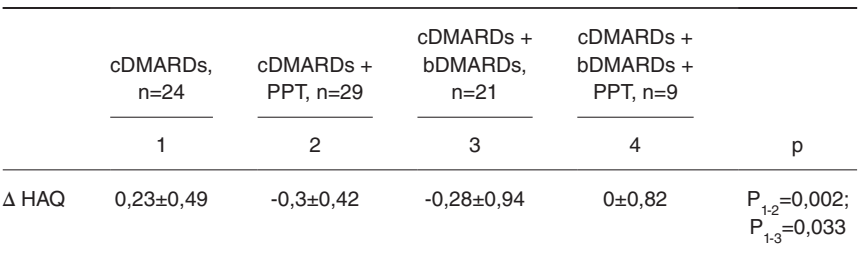

Conclusion: Functional abilities measured by HAQ scores significantly improved in RA-patients with ADD receiving cDMARDs in combination with bDMARDs or PPT compared to cDMARDs only. The lowest HAQ scores were observed in patients receiving cDMARDs in combination with PPT.

Disclosure of Interests: None declared

DOI: 10.1136/annrheumdis-2020-eular.367

\section{Rheumatoid arthritis - prognosis, predictors and outcome II}

\begin{tabular}{|l|l}
\hline OP0216 & DEVELOPMENT AND VALIDATION OF PATIENT-LEVEL \\
PREDICTION MODELS FOR ADVERSE HEALTH \\
OUTCOMES AMONGST ADULT RA PATIENTS INITIATING \\
FIRST-LINE TREATMENT OF METHOTREXATE \\
MONOTHERAPY: A MULTINATIONAL REAL-WORLD \\
COHORT ANALYSIS INCLUDING 164,735 SUBJECTS
\end{tabular}

C. Yang ${ }^{1}$, R. Williams ${ }^{2}$, J. Swerdel ${ }^{3}$, M. Jani ${ }^{4}$, T. Duarte-Salles ${ }^{5}$,

K. Chatzidionysiou ${ }^{6}$, D. Prieto-Alhambra ${ }^{7}$, P. Ryan ${ }^{8}$, P. Rijnbeek ${ }^{9}$ on behalf of 'European Health Data and Evidence Network (EHDEN) RA Research Group. ' Department of Medical Informatics EMC, Rotterdam, Netherlands; ${ }^{2}$ Department of Medical Informatics Erasmus MC, Rotterdam, Netherlands; ${ }^{3} J a n s s e n$ Research and Development, New Jersey, United States of America; ${ }^{4}$ Centre for Epidemiology Versus Arthritis, University of Manchester, Manchester, United Kingdom; ${ }^{5}$ Fundació Institut Universitari per a la Recerca a l'Atenció Primària de Salut Jordi Gol i Gurina (IDIAPJGol), Barcelona, Spain; ${ }^{6}$ Dep of Medicine, Solna, Rheum Unit, Karolinska Institutet, Stockholm, Sweden; ${ }^{7}$ University of Oxford, NDORMS, Oxford, United Kingdom; ${ }^{8}$ Johnson \& Johnson, New Jersey, United States of America; ${ }^{9}$ Erasmus University, Department of Medical Informatics, Rotterdam, Netherlands

Background: EULAR guidelines recommend the early initiation of methotrexate (MTX) monotherapy as soon as possible after the diagnosis of rheumatoid arthritis (RA). Evaluating patient-level risks for adverse outcomes after MTX initiation would allow clinicians to provide more personalised care.

Objectives: To develop and validate patient-level prediction models for adverse health outcomes including leukopenia, pancytopenia, infection (serious, opportunistic, all), cardiovascular disease (CVD) (myocardial infarction (MI), stroke), and cancer (breast, colorectal, uterus) in adult RA patients initiating first-line treatment of MTX monotherapy

Methods: Health data from claims and electronic health records were used including patients from 7 European countries (Spain, Estonia, Netherlands, Belgium, Germany, France, and the UK), the United States of America, Australia, and Japan. All RA patients initiating first-line treatment of MTX monotherapy with at least one year of prior observation were included. Prediction models for the outcomes were developed for a time at risk of 3 months (infections, leukopenia, pancytopenia), 2 years ( $\mathrm{Ml}$ and stroke), and 5 years (cancers) on the Optum $\odot$ De-Identified Clinformatics $®$ Data Mart Database. Models were developed using LASSO logistic regression and were evaluated using the area under the receiver operator characteristic curve (AUROC) for discrimination and graphically assessed for calibration. The models were externally validated on all other databases.

Results: A total of 21,307 subjects were used for training and validated against 143,427 patients from 14 sites. MI (AUROC internal 0.77, AUROC external ranging from 0.49 to 0.78 ), stroke (AUROC internal 0.78 , AUROC external ranging from 0.68 to 0.79 ) and serious infection (AUROC internal 0.75 , AUROC external ranging from 0.63 to 0.79 ) had good predictive validity [Table 1]. Discrimination for all other outcomes was lower, with al AUC $<0.7$ in internal validation. For detailed results see: https://data.ohdsi. org/ehdenRaPrediction/

Table 1. Internal (Optum) and external validation results: AUC ROC for discrimination

\begin{tabular}{lccc}
\hline Database & Acute Ml within 2y & Stroke within 2y & Serious Infection within 3m \\
\hline Optum (internal) & 0.77 & 0.78 & 0.75 \\
PanTher & 0.76 & 0.78 & 0.74 \\
IQVIA_AMBEMR & 0.76 & 0.72 & 0.66 \\
CCAE & 0.73 & 0.73 & \\
IQVIA_GERMANY & 0.64 & 0.70 & 0.67 \\
IQVIA_THIN & 0.62 & 0.65 & 0.61 \\
MDCR & 0.68 & 0.68 & 0.63 \\
IQVIA_HOSPITAL & 0.67 & 0.63 & 0.71 \\
MDCD & 0.72 & 0.79 & 0.82 \\
JMDC & 0.49 & 0.75 & \\
IQVIA_LPDFRANCE & 0.69 & & \\
Estonia & 0.67 & 0.77 & \\
IQVIA_AUS & 0.58 & & \\
IPCI & 0.68 & 0.75 & \\
SIDIAP & 0.65 & & \\
\end{tabular}

Conclusion: Clinical tools were developed that successfully identify subjects at risk of MI, stroke and serious infection at the initiation of first-line MTX therapy The developed algorithms had good transportability and generally, the models with high AUROC had adequate internal calibration although some external validations show they could benefit from recalibration. For short-term opportunistic and all infections, as well as 5-year cancer models, we were unable to achieve a high enough AUROC to warrant validating externally.

Disclosure of Interests: Cynthia Yang: None declared, Ross Williams: None declared, Joel Swerdel Shareholder of: J\&J shares, Grant/research support from: Full-time employment salary from Janssen, Consultant of: Janssen employee, Employee of: Janssen, Paid instructor for: Janssen employee, have instructed at conferences, Speakers bureau: Janssen employee, have spoken at conferences, Meghna Jani Speakers bureau: Grifols, Talita Duarte-Salles: None declared, Katerina Chatzidionysiou Consultant of: AbbVie, Pfizer, Lilly., Daniel Prieto-Alhambra Grant/research support from: Professor Prieto-Alhambra has received research Grants from AMGEN, UCB Biopharma and Les Laboratoires Servier, Consultant of: DPA's department has received fees for consultancy services from UCB Biopharma, Speakers bureau: DPA's department has received fees for speaker and advisory board membership services from Amgen, Patrick Ryan: None declared, Peter Rijnbeek: None declared

DOI: 10.1136/annrheumdis-2020-eular.3606

\begin{tabular}{|l|l|}
\hline OP0217 & INVOLVEMENT OF LARGE JOINTS AT DISEASE \\
& PRESENTATION IS ASSOCIATED WITH DIVERSE \\
& HISTOPATHOLOGICAL FEATURES AND CLINICAL \\
& OUTCOMES IN EARLY RHEUMATOID ARTHRITIS
\end{tabular}

F. Rivellese $^{1}$, F. Humby ${ }^{1}$, G. Lliso Ribera ${ }^{1}$, A. Nerviani ${ }^{1}$, E. Sciacca ${ }^{1}$, G. Giorli ${ }^{1}$, R. Hands ${ }^{1}$, L. Fossati-Jimack ${ }^{1}$, G. Thorborn ${ }^{1}$, M. Lewis ${ }^{1}$, C. Pitzalis ${ }^{1}$.

${ }^{1}$ Queen Mary University of London, Centre for Experimental Medicine and Rheumatology, London, United Kingdom

Background: The involvement of large joints at disease presentation in early Rheumatoid Arthritis (RA) has been associated with severe disease activity. A the same time, the clinical heterogeneity of RA is known to be mirrored by heterogeneity of synovial inflammation, with specific histological patterns (pathotypes) 
associated with treatment response and disease progression. However, it is not known whether joint size is associated with specific pathotypes.

Objectives: To analyse histopathological features of synovial biopsies from joints of different sizes and establish the relationship with clinical outcomes in patients with early RA.

Methods: 167 patients with early ( $<1$ year) treatment-naïve RA, fulfilling the 2010 RA criteria and recruited at Barts Health NHS Trust, underwent US-guided synovial biopsy of the most inflamed joint, either large (knee), medium (e.g. wrist, ankle, elbow) or small (MCPs, MTPs), before starting treatment with csDMARDS with a treat to target approach. Upon SQ scoring (0-4) of immune cell infiltration, tissues were classified into lympho-myeloid, diffuse-myeloid and pauci-immune pathotypes. Synovial samples from 111 patients underwent RNA-seq.

Results: The majority of synovial biopsies were performed on medium and small joints $(60.6 \%$ and $19.4 \%)$ as compared to $21.3 \%$ in large joints (Table 1). At baseline, patients who underwent large joint biopsy showed significantly higher levels of inflammation (CRP 27.9 \pm 32.4 large, 20.7 \pm 26.9 medium, $10.4 \pm 9.8$ small, $p=0.007)$ and higher $\mathrm{HAQ}(1.8 \pm 0.7$ large, $1.4 \pm$ 0.8 medium, $1.2 \pm 0.9$ small, $p=0.012$ ), with no differences in DAS28. Significantly higher inflammatory scores and higher proportion of lympho-myeloid pathotype were observed in large joints (Table 1 and Figure 1). 6 months after treat-to-target treatment with csDMARDs, large joints patients had significantly higher $\mathrm{HAQ}$ and lower response (RR for low disease activity in large vs medium joints $0.5,95 \% \mathrm{Cl} 0.2-0.9, \mathrm{p}=0.03$ ). Finally, differentially expressed genes by RNA-seq showed segregation according to joint size (Figure 2), with upregulation of genes of the Homeobox transcription factors family in large joints.

Table 1. EULAR 2010 RA $(n=167)$

\begin{tabular}{|c|c|c|c|c|c|}
\hline & & $\begin{array}{c}\text { Large joints\# } \\
33(19.4 \%)\end{array}$ & $\begin{array}{c}\text { Medium joints\# } \\
100(60.6 \%)\end{array}$ & $\begin{array}{l}\text { Small Joints\# } \\
34(20 \%)\end{array}$ & $P^{*}$ \\
\hline \multicolumn{6}{|l|}{ Clinical features } \\
\hline ESR $\mathrm{mm} / \mathrm{h}$, mean (SD) & & $48.2(31.5)$ & $39.6(30.8)$ & $29.2(17.3)$ & ns \\
\hline CRP mg/L, mean (SD) & & $27.9(32.4)$ & $20.7(26.9)$ & $10.4(9.8)$ & 0.007 \\
\hline DAS28, mean (SD) & & $6(1.2)$ & $5.7(1.4)$ & $5.7(1.5)$ & ns \\
\hline HAQ, mean (SD) & & $1.8(0.7)$ & $1.4(0.8)$ & $1.2(0.9)$ & 0.012 \\
\hline ACPA-positive, $\%$ & & $70.9 \%$ & $77.3 \%$ & $83.9 \%$ & ns \\
\hline RF-positive, \% & & $71.9 \%$ & $74.2 \%$ & $80.6 \%$ & ns \\
\hline \multicolumn{6}{|l|}{ Histology } \\
\hline $\begin{array}{l}\text { Inflammatory score, } \\
\text { median IQR) }\end{array}$ & & $5(3)$ & $4(4)$ & $2(2.75)$ & $<0.001$ \\
\hline \multirow{4}{*}{ Pathotype, \% } & Ungraded & $6.1 \%$ & $7.8 \%$ & $2.9 \%$ & 0.014 \\
\hline & Fibroid & $6.1 \%$ & $24.3 \%$ & $32.3 \%$ & \\
\hline & Myeloid & $30.3 \%$ & $28.1 \%$ & $47.1 \%$ & \\
\hline & $\begin{array}{l}\text { Lympho- } \\
\text { myeloid }\end{array}$ & $57.6 \%$ & $39.8 \%$ & $17.6 \%$ & \\
\hline
\end{tabular}

Clinical outcomes at 6 months

\begin{tabular}{lcccc}
\hline DAS28 6m, mean (SD) & $4.2(1.8)$ & $3.4(1.9)$ & $3.7(1.5)$ & $\mathrm{ns}$ \\
HAQ $6 \mathrm{~m}$, mean (SD) & $1.2(0.8)$ & $0.8(0.8)$ & $0.8(0.8)$ & 0.012 \\
DAS28 6m <3.2, \% & $23.3 \%$ & $48.8 \%$ & $37.9 \%$ & 0.04
\end{tabular}

\#Large joints: knees; Medium joints: wrists, ankle, elbows; Small joints: MCPs, MTPs, PIPs; * Chi-squared or Kruskal-Wallis as appropriate;

Conclusion: Synovial biopsy of large joints as the most inflamed joints at disease presentation identified patients with early RA with specific histopathological features and clinical outcomes. Together with clustering of differentially expressed genes according to joint size, this suggests that the involvement of different joint compartments in early RA contributes to disease heterogeneity with potential physiopathological and clinical implications.

References:

[1] Humby et al Ann Rheum Dis. 2019 Jun;78(6):761-772

[2] Lewis et al Cell Rep. 2019 Aug 27;28(9):2455-2470.e5

[3] Linn-Rasker SP et al Ann Rheum Dis. 2007 May;66(5):646-50

Acknowledgments: PEAC http://www.peac-mrc.mds.qmul.ac.uk MRC grant 36661 \& ARUK Grant 20022

F. Rivellese NIHR Fellowship TRF-2018-11-ST2-002
Figure 1
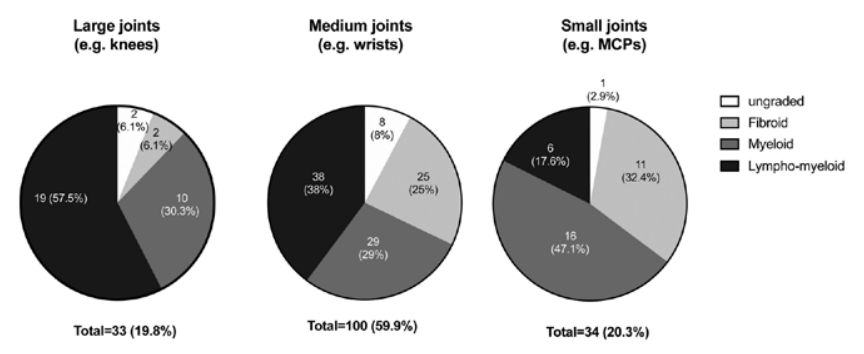

Figure 2

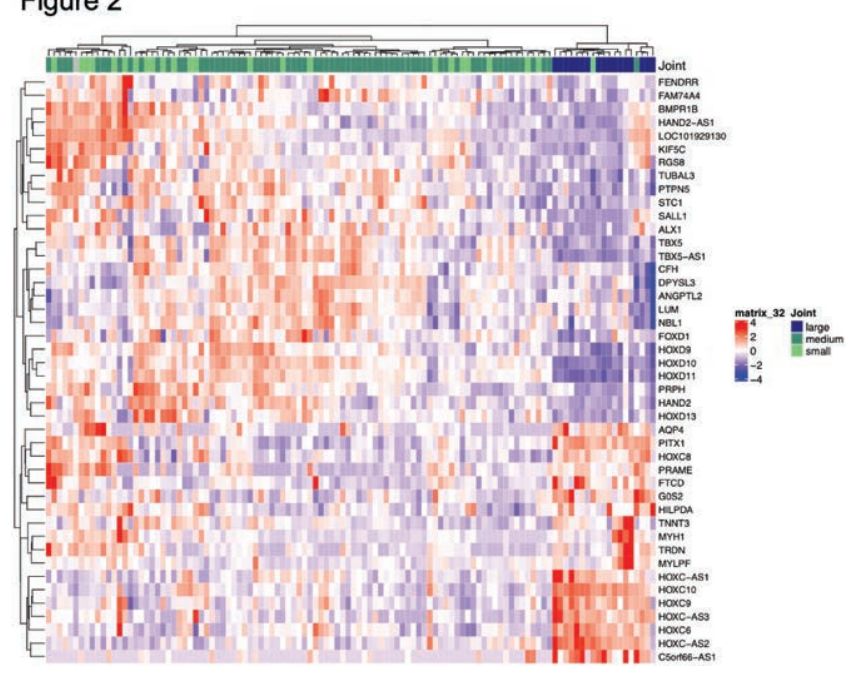

Disclosure of Interests: None declared

DOI: 10.1136/annrheumdis-2020-eular.2475

\section{OP0218 \\ CENTRAL NERVOUS SYSTEM PAIN RESPONSE AND COMPONENTS OF DISEASE ACTIVITY IN RA PATIENTS AFTER TREATMENT WITH CERTOLIZUMAB OR PLACEBO: A POST-HOC ANALYSIS FROM THE PRECEPRA TRIAL}

H. Schenker ${ }^{1}$, J. Rech ${ }^{1}$, K. Tascilar ${ }^{1}$, M. Hagen ${ }^{1}$, V. Schönau ${ }^{1}$, M. Sergeeva ${ }^{2}$, M. Selvakumar', L. Konerth ${ }^{2}$, J. Prade ${ }^{2}$, S. Strobelt ${ }^{2}$, L. Valor ${ }^{1}$, A. Hueber ${ }^{3}$, D. Simon ${ }^{1}$, A. Kleyer ${ }^{1}$, F. Behrens ${ }^{4}$, J. A. P. Da Silva ${ }^{5}$, C. Baerwald ${ }^{6}$, S. Finzel ${ }^{7}$, R. Voll ${ }^{7}$, E. Feist ${ }^{8,9}$, A. Doerfler ${ }^{10}$, N. Damjanov ${ }^{11}$, A. Hess ${ }^{2}$, G. Schett ${ }^{1}{ }^{1}$ FriedrichAlexander University (FAU) Erlangen-Nürnberg and Universitätsklinikum Erlangen, Department of Internal Medicine 3 - Rheumatology and Immunology, Erlangen, Germany; ${ }^{2} F A U$ Erlangen-Nürnberg, Institute for Experimental Pharmacology, Erlangen, Germany; ${ }^{3}$ Sozialstiftung Bamberg, Section Rheumatology, Bamberg, Germany; ${ }^{4}$ Goethe University Frankfurt, Rheumatology and Fraunhofer TMP, Frankfurt am Main, Germany; ${ }^{5}$ Hospitais da Universidade (SRHUC), Department, Coimbra, Portugal; ${ }^{6}$ Universität Leipzig, Medizinische Klinik III - Bereich Rheumatologie, Leipzig, Germany; ${ }^{7}$ Universitätsklinikum Freiburg, Klinik für Rheumatologie und Klinische Immunologie, Freiburg, Germany; ${ }^{8}$ Klinik für Rheumatologie der Helios Fachklinik, Vogelsang-Gommern, Germany; ${ }^{9}$ Charité - Universitätsmedizin Berlin, Dpt. of Rheumatology and Clinical Immunology, Berlin, Germany; ${ }^{10}$ Friedrich-Alexander University (FAU) Erlangen-Nürnberg and Universitätsklinikum Erlangen, Abteilung für Neuroradiologie, Erlangen, Germany; ${ }^{11}$ Belgrade University School of Medicine, Institute of Rheumatology, Belgrade, Serbia

Background: We have previously observed in RA patients that central nervous system (CNS) response to compression of a painful joint, measured using functional MRI (fMRI) of the brain as the number of blood oxygen level dependent (BOLD) signal positive voxels, is rapidly ameliorated, much earlier than any clinical response with anti-TNF treatment and a high baseline CNS pain response could predict better response to certolizumab pegol (CZP) treatment. Pre-CePRA was designed and conducted to test this effect in a randomized, placebo controlled trial of CZP and showed an incremental linear trend of DAS28 low disease activity (LDA) across study groups treated with placebo, and two CZP arms stratified as low or high pre-treatment CNS pain response. 